\title{
Impact of sperm DNA fragmentation on clinical in vitro fertilization outcomes
}

\author{
Hwa Young Choi ${ }^{1,2}$, Seul Ki Kim ${ }^{2,3}$, Seok Hyun Kim ${ }^{2,4}$, Young Min Choi ${ }^{2,4}$, Byung Chul Jee ${ }^{2,3}$ \\ 'Department of Obstetrics and Gynecology, Maria Fertility Hospital, Seoul; ${ }^{2}$ Department of Obstetrics and Gynecology, Seoul National University \\ College of Medicine, Seoul; ${ }^{3}$ Department of Obstetrics and Gynecology, Seoul National University Bundang Hospital, Seongnam; ${ }^{4}$ Department of \\ Obstetrics and Gynecology, Seoul National University Hospital, Seoul, Korea
}

Objective: We studied the association between sperm DNA fragmentation (SDF) and several clinical in vitro fertilization outcomes.

Methods: We retrospectively analyzed 169 consecutive fresh IVF cycles. Semen was collected on the day of oocyte retrieval, and we assessed standard semen parameters and the SDF level (by terminal deoxynucleotidyl transferase dUTP nick-end labeling). Poor ovarian response (POR) was defined as the collection of three or fewer mature oocytes. Oocytes were inseminated by the conventional method or intracytoplasmic sperm injection.

Results: SDF did not affect the fertilization or pregnancy rate, but did have a significant effect on the miscarriage rate. In the miscarriage group $(n=10)$, the SDF level was significantly higher (23.9\% vs. $14.1 \%)$ and number of mature oocytes was significantly lower (4.3 vs. 7.6$)$ than in the live birth group $(n=45)$. Multiple regression analysis showed that SDF was an independent predictor of miscarriage (odds ratio, 1.051; 95\% confidence interval, 1.001-1.104). The cutoffs for the SDF level and number of mature oocytes that could predict miscarriage were $>13 \%$ and $\leq 3$, respectively. In the low-SDF group $(\leq 13 \%)$, the miscarriage rate was similar in POR patients and those with a normal ovarian response (NOR; $14.2 \%$ vs. $4.3 \%$ ). In the high-SDF group ( $>13 \%$ ), the miscarriage rate was significantly higher in the POR group than in the NOR group (60.0\% vs. $13.3 \%, p=0.045)$.

Conclusion: Our study demonstrated that a high SDF level ( $>13 \%$ ) was associated with a high miscarriage rate, and that it mainly contributed to miscarriage in the POR group. The results suggest that SDF measurements should be considered in couples with POR in order to predict the prognosis of the pregnancy.

Keywords: Abortion; DNA fragmentation; Fertilization in vitro; Pregnancy; Spermatozoa

\section{Introduction}

Sperm DNA fragmentation (SDF) is not routinely assessed as part of semen analysis, but it can be offered as a special test [1]. Debate continues on whether SDF measurements should become a part of the

Received: Oct 4, 2017 · Accepted: Oct 26, 2017

Corresponding author: Byung Chul Jee

Department of Obstetrics and Gynecology, Seoul National University Bundang

Hospital, 82 Gumi-ro 173beon-gil, Bundang-gu, Seongnam 13620, Korea

Tel: +82-31-787-7254 Fax: +82-31-787-4054 E-mail: blasto@snubh.org

*This work was supported by the Korea Health Care Technology R\&D Project, Ministry of Health and Welfare, Korea (No. A120043).

This is an Open Access article distributed under the terms of the Creative Commons Attribution Non-Commercial License (http://creativecommons.org/licenses/by-nc/4.0/) which permits unrestricted non-commercial use, distribution, and reproduction in any medium, provided the original work is properly cited. routine fertility workup [2]. A number of studies have evaluated the influence of SDF levels on fertilization, embryo quality, and pregnancy outcomes, and five meta-analyses have been published [3-7].

Based on the high-quality evidence from the meta-analyses, high SDF levels appear to be associated with lower rates of clinical pregnancy in standard in vitro fertilization (IVF) cycles, but not in intracytoplasmic sperm injection (ICSI) cycles [3,5,6]. A significantly lower live birth rate in patients with a high SDF level has been reported, and this negative impact was prominent in IVF cycles [7]. Moreover, high SDF levels appear to be associated with higher miscarriage rates in ICSI cycles only [5] or in overall cycles [4], although some studies have reported no significant association with miscarriage rates [6]. Regarding the fertilization rate, one meta-analysis reported no association with SDF levels in either standard IVF or ICSI cycles [3]. 
Although positive associations between SDF levels and several IVF outcomes have been reported, the majority of studies have not reported a clear cutoff SDF level predictive of poor fertilization, clinical pregnancy, live birth, or miscarriage rates.

When investigating the association of SDF levels with fertilization rates, previous studies have divided cycles into standard IVF groups and ICSI groups. However, since the indications for ICSI include not only male factors but also certain female factors, ICSI groups should be divided into male and female factor groups and evaluated separately.

In this study, we investigated the associations between SDF levels and several IVF outcomes, such as the fertilization rate, pregnancy rate, and miscarriage rate. For the associations between SDF levels and the fertilization rate, separate analyses were performed for standard IVF patients and those who underwent ICSI due to male or female factors, respectively.

\section{Methods}

\section{Study population}

The dataset for this retrospective study included 169 consecutive fresh IVF cycles performed between January 2012 and June 2014 at the Seoul National University Bundang Hospital. This study was approved by the Institutional Review Board of the Seoul National University Bundang Hospital (IRB No. B-1608-357-108). Written informed consents were obtained. The indications for IVF were unexplained infertility $(n=78)$, tubal factor infertility $(n=26)$, age factor infertility $(n=20)$, endometriosis $(n=18)$, uterine factor infertility $(n=10)$, polycystic ovary syndrome $(n=9)$, and male factor infertility $(n=8)$. The body mass index, basal serum level of follicle-stimulating hormone (FSH), and a random serum level of anti-Müllerian hormone in the female were recorded if they were measured within 2 months before starting the cycle.

\section{Stimulation protocols and oocyte collection}

Controlled ovarian stimulation was performed using recombinant FSH (Gonal-F; Serono, Geneva, Switzerland) with or without highly purified urinary gonadotropin (Menopur; Ferring, Malmo, Sweden) using a luteal long protocol with a gonadotropin-releasing hormone $(\mathrm{GnRH})$ agonist $(0.1 \mathrm{mg} /$ day of Decapeptyl; Ferring) $(\mathrm{n}=10)$ or a $\mathrm{GnRH}$ antagonist protocol ( $0.25 \mathrm{mg} /$ day of Cetrotide, Serono) $(n=157)$. When two or more leading follicles reached a mean diameter of $\geq 18 \mathrm{~mm}, 250 \mu \mathrm{g}$ of recombinant human chorionic gonadotropin (hCG; Ovidrel, Serono) was injected. Oocytes were retrieved 36 hours after the hCG injection. Poor ovarian response (POR) was defined as the collection of three or fewer mature oocytes. Metaphase I-derived in vitro matured oocytes were counted as mature oocytes, but germinal vesicle-derived oocytes were not.

\section{Semen collection, measurement of SDF, and in vitro fertilization}

Semen was collected on the day of oocyte retrieval and standard sperm quality was assessed in the raw semen (concentration, motility, and normal form using strict criteria). Sperm quality was defined as normal $(n=104)$ when the semen parameters were within the World Health Organization (WHO) reference values, regardless of the patient's diagnosis. The subnormal group $(n=43)$ included semen with parameters outside the WHO criteria, but not to the point of requiring ICSI, and the abnormal group $(n=22)$ was made up of cases requiring ICSI.

The SDF level was measured by the terminal deoxynucleotidyl transferase dUTP nick-end labeling (TUNEL) method in the raw semen, following our previously reported institutional protocol [8]. Semen samples were smeared on a silane-coated slide (DAKO Japan Co., Tokyo, Japan) and air-dried. Samples were fixed with $4 \%$ paraformaldehyde for 1 hour at $15^{\circ} \mathrm{C}-25^{\circ} \mathrm{C}$, washed with phosphate-buffered saline, and then were permeabilized with $0.1 \%$ Triton X-100 in $0.1 \%$ sodium citrate (Sigma-Aldrich, St. Louis, MO, USA). A commercial apoptosis detection kit (in situ cell death detection kit; Roche Diagnostics, Mannheim, Germany) was used to assess cell death in the samples according to the manufacturer's instructions. Counterstaining was performed using a mounting medium with 4,6-diamidino2-phenylindole (DAPI; Vector Laboratories, Burlingame, CA, USA). The nuclei of sperm with fragmented DNA was stained green, whereas the nuclei of other cells were stained blue. Sperm heads with $>50 \%$ of the area stained green were considered positive for DNA fragmentation, following our previously reported institutional protocol [8]. At least 500 sperm cells were counted per experimental set, and the SDF level was determined as the percentage of sperm with fragmented DNA.

The remaining semen was processed by a discontinuous gradient as described in the kit instructions (Sydney IVF density gradient media; COOK, Brisbane, Australia). After initial centrifugation of the semen ( $300 \times g$ for 5 minutes) to remove the seminal plasma, the obtained pellet was suspended in fresh Ham's F10 medium (1.5 mL) supplemented with $10 \%$ synthetic serum substitute (SSS; Irvine Scientific, Santa Ana, CA, USA). Pre-washed semen (1.0 mL) was layered on the top of a discontinuous gradient in a $15-\mathrm{mL}$ conical tube $(40 \% / 80 \%)$. The conical tube was centrifuged at $300 \times g$ for 5 minutes, and the sperm cells collected from the bottom layer ( $80 \%$ layer) were washed twice by resuspension in $4 \mathrm{~mL}$ of Ham's F10 medium and centrifugation $(300 \times g$ for 5 minutes). After two rounds of centrifugation, the supernatant was removed and the pellet was resuspended in $3 \mathrm{~mL}$ of Ham's F10 medium supplemented with 10\% SSS. The resuspended pellet was later used in insemination.

The oocytes were inseminated by the conventional method 
( $n=69)$, by ICSI $(n=95)$, or by split insemination $(n=3)$, depending on the quality of the sperm and oocyte. ICSI was used in 22 cycles due to a male factor and in 73 cycles due to a female factor. Fertilization was confirmed by observing a two-pronuclear zygote on the day after oocyte retrieval. A low fertilization rate was defined as $\leq 75 \%$ in the current study, which corresponded to the 25th percentile of the overall fertilization rate.

\section{Embryo transfer and confirmation of pregnancy}

The embryos were transferred 3 or 5 days after oocyte retrieval. Embryo quality was evaluated by morphological criteria based on the degree of fragmentation and the regularity of blastomeres on day 3 after fertilization. The embryos were graded as follows: grade A, $0 \%$ anucleate fragments, regularity of blastomeres, and no apparent morphological abnormality; grade B, <20\% anucleate fragments, regularity of blastomeres, and no apparent morphological abnormality; grade C, $20 \%-50 \%$ anucleate fragments, irregularity of blastomeres, and no apparent morphological abnormality; and grade $D$, $>50 \%$ anucleate fragments, irregularity of blastomeres, and apparent morphological abnormalities. Blastocysts were evaluated on day 5 by the developmental stage and quality of the inner cell mass and trophectoderm. A good-quality blastocyst was defined as grade $A A$, $A B, A C, B A, B B$, or $C A$. Luteal phase support was performed using either a daily dose of $50 \mathrm{mg}$ of progesterone in oil (Progest; Genefer, Seoul, Korea) or an 8\% progesterone gel (Crinone, Serono), starting on the day of oocyte retrieval. Pregnancy was first assessed 14 days after oocyte retrieval by measuring the serum hCG level. In cases with positive hCG results, transvaginal ultrasonography was performed to confirm an intrauterine pregnancy and to identify the number of gestational sacs and the fetal heartbeat. Clinical pregnancy was defined as the presence of one or more gestational sacs. Miscarriage was defined as pregnancy loss before 12 weeks of gestation.

\section{Statistical analysis}

All statistical analyses were performed using SPSS PASW ver. 18.0 (SPSS Inc., Chicago, IL, USA). When analyzing the association between standard sperm quality and SDF levels, the data from 169 sperm samples were used. The data from 164 cycles were used to analyze the fertilization rate, after excluding three cases with split insemination and two cases with no mature oocyte. Associations between SDF levels and pregnancy were analyzed in the 157 cycles in which embryo transfer was performed. Associations between SDF levels and miscarriage were analyzed in the 55 cases that achieved clinical pregnancy. Correlations were tested using the non-parametric Spearman rank test. The chi-square test was used to compare proportions between two groups. If the cell numbers were $<5$, the Fisher exact test was applied to compare frequencies between groups. The medians of numeric data were compared using the Mann-Whitney U-test. Multiple regression analyses were performed for several numeric variables. A receiver operating characteristic (ROC) curve analysis was used to assess specific cutoff values for several numeric parameters. Results were considered significant with a two-tailed $p$-value $<0.05$.

\section{Results}

\section{SDF levels and standard sperm parameters}

The SDF levels ranged from $0.4 \%$ to $56.8 \%$ in the 169 sperm samples (mean, 15.1\%; median, 11.8\%; standard deviation, 12.2\%). They did not show a normal distribution. As shown in Table 1, in the overall population, a positive correlation between SDF and the male partner's age was found, but an inverse correlation was found between SDF and sperm motility. A multivariate analysis revealed that both the male partner's age and motility were significantly associated with SDF $(S D F=-0.444+[0.618 \times$ male partner's age $]-[0.162 \times$ motility]). The positive correlation between SDF and the male partner's age was prominent in those with normal sperm, while an inverse correlation between SDF and motility was prominent in the group with subnormal sperm. In the group with abnormal sperm, no significant correlations were found between SDF and any of the standard sperm parameters.

In the group with normal sperm, the median SDF level (95\% confidence interval $[\mathrm{Cl}])$ was $11.0 \%(8.5 \%-13 \%)$. The median SDF level

Table 1. Correlation coefficients for associations of the sperm DNA fragmentation level with standard sperm parameters

\begin{tabular}{lcccc}
\hline \multirow{2}{*}{ Variable } & Overall $(\mathrm{n}=169)$ & \multicolumn{3}{c}{ Sperm quality } \\
\cline { 3 - 5 } & & Normal $(\mathrm{n}=104)$ & Subnormal $(\mathrm{n}=43)$ & Abnormal $(\mathrm{n}=22)$ \\
\hline Age of male partner $(\mathrm{yr})$ & $0.29^{\mathrm{a})}$ & $0.31^{\mathrm{a}}$ & 0.20 & 0.38 \\
Volume (mL) & -0.14 & -0.09 & 0.04 & -0.25 \\
Concentration (million/mL) & 0.06 & 0.01 & $0.33^{\mathrm{a})}$ & 0.27 \\
Motility (\%) & $-0.21^{\mathrm{a})}$ & -0.06 & $-0.53^{\mathrm{a})}$ & -0.31 \\
Total motile sperm (million) & -0.13 & -0.08 & -0.09 & -0.14 \\
Normal form (\%) & 0.07 & 0.01 & 0.22 & -0.02 \\
\hline
\end{tabular}

${ }^{\text {a) }} p<0.05$ by the Spearman rank test. 

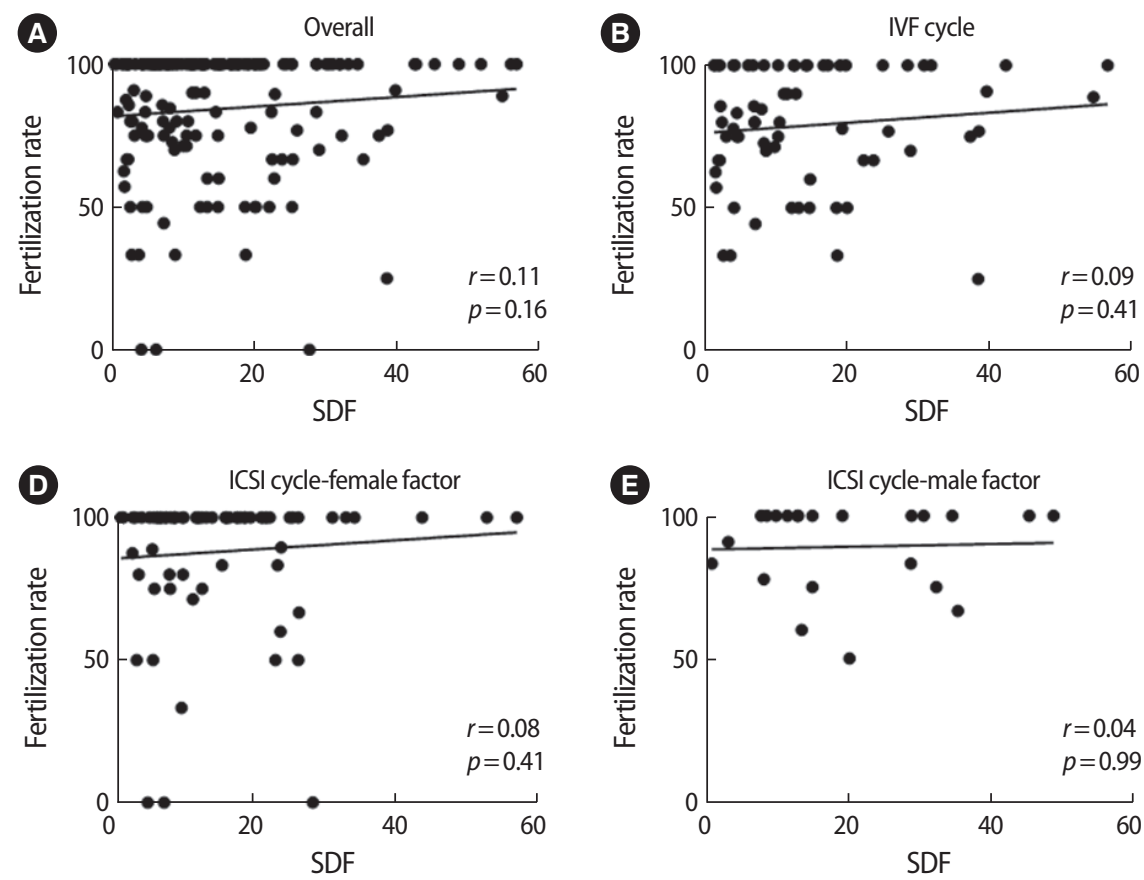

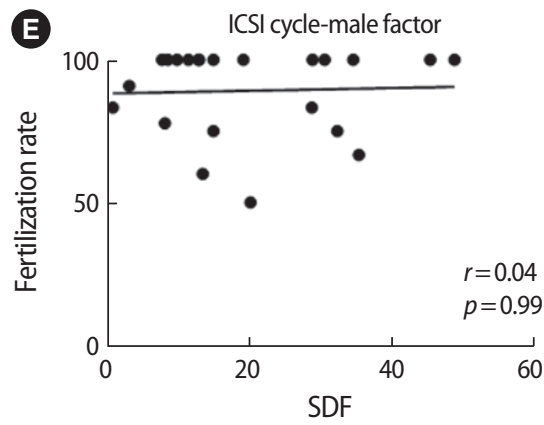

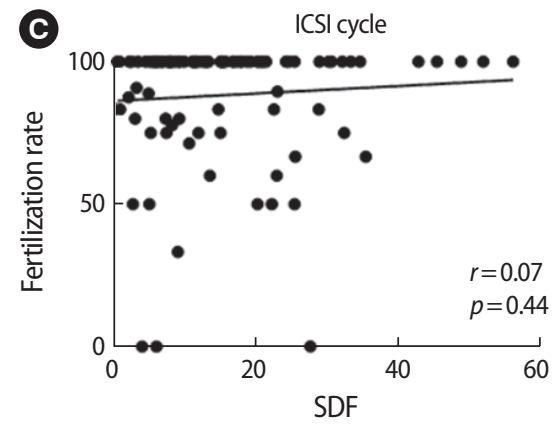

Figure 1. Associations between sperm DNA fragmentation (SDF) levels and the fertilization rate in the overall study population and in subgroups defined according to the insemination method or whether intracytoplasmic sperm injection (ICSI) was performed due to a female or male factor ( $r$ : correlation coefficients by the Spearman rank test). (A) Overall group, (B) conventional in vitro fertilization (IVF) group, (C) ICSI group, (D) ICSI due to female factor, (E) ICSI due to male factor.

Table 2. Association between the sperm DNA fragmentation level and the fertilization rate in the overall study population and sub-groups defined according to sperm quality

\begin{tabular}{|c|c|c|c|c|c|c|c|c|c|}
\hline \multirow{3}{*}{ Variable } & \multicolumn{9}{|c|}{ Sperm quality } \\
\hline & \multicolumn{3}{|c|}{ Normal } & \multicolumn{3}{|c|}{ Subnormal } & \multicolumn{3}{|c|}{ Abnormal } \\
\hline & $\mathrm{FR}(\%)$ & No. & $r$ & $\mathrm{FR}(\%)$ & No. & $r$ & $\mathrm{FR}(\%)$ & No. & $r$ \\
\hline Overall & 100 & 102 & -0.02 & 87.3 & 40 & 0.28 & 100 & 22 & 0.04 \\
\hline IVF group & 84 & 50 & 0.05 & 75 & 19 & 0.27 & & & \\
\hline ICSI group-female factor & 100 & 52 & -0.09 & 100 & 21 & 0.27 & & & \\
\hline ICSI group-male factor & & & & & & & 100 & 22 & 0.04 \\
\hline
\end{tabular}

The median values for FR are given. Spearman rank test.

FR, fertilization rate; IVF, in vitro fertilization; ICSI, intracytoplasmic sperm injection.

was $13.0 \%(7.2 \%-18.7 \%)$ in the group with subnormal sperm and $14.9 \%(10.4 \%-29.8 \%)$ in the group with abnormal sperm. There was a significant difference between the median SDF level between the normal and abnormal sperm groups $(p<0.05)$.

\section{Impact of SDF on fertilization rate}

SDF levels were not correlated with the fertilization rate in the overall study population or in subgroups defined according to the insemination method or whether ICSI was performed due to a male or female factor (Figure 1). In the three subgroups defined according to sperm quality, no significant correlations were found between SDF and the fertilization rate in the overall population, the standard IVF group, or the ICSI group with a female or male factor (Table 2).

A ROC analysis was performed to evaluate whether SDF levels could predict a low fertilization rate (i.e., $\leq 75 \%$ ). The cutoff value for the SDF level was $\leq 5.1 \%$ in the overall population, $\leq 4.9 \%$ in the standard IVF group, $>21.3 \%$ in the ICSI group, $>21.3 \%$ in the female factor ICSI group, and > 12.9\% in the male factor ICSI group. However, none of the values were statistically significant.

\section{Impact of SDF on clinical pregnancy}

The clinical pregnancy rate was 31\% (40/129) in the day 3 transfer group and 53.5\% (15/28) in the day 5 transfer group. SDF levels and other standard sperm parameters did not differ according to whether pregnancy was achieved (data not shown). Two factors were significantly associated with pregnancy: female age (35 vs. 37 years, $p=0.016)$ in the day 3 transfer group and the male partner's age (34 vs. 38 years) in the day 5 transfer group ( $p=0.002$ ).

\section{Impact of SDF on miscarriage}

Clinical pregnancy was achieved in 55 women, but 10 pregnancies ended in spontaneous miscarriage. In the miscarriage group, the SDF levels and serum FSH levels were significantly higher than in the live birth group, and the number of mature oocytes was significantly 
Table 3. Comparison between the live birth group and the miscarriage group

\begin{tabular}{|c|c|c|c|}
\hline Variable & Live birth $(n=45)$ & Miscarriage $(n=10)$ & $p$-value \\
\hline Age of male partner (yr) & $36.8(35.4-38.3)$ & $37.8(34.6-41.1)$ & NS \\
\hline Volume (mL) & $3.1(2.7-3.5)$ & $3.2(2.5-3.9)$ & NS \\
\hline Concentration (million/mL) & $133(93-172)$ & $135(92-177)$ & NS \\
\hline Motility (\%) & $51(45.9-56.0)$ & $55.9(41.8-70.0)$ & NS \\
\hline Total motile sperm count (million) & $184(128-240)$ & $214(144-284)$ & NS \\
\hline Normal form (\%) & $10.1(8.2-11.9)$ & $10.6(6.2-15.0)$ & NS \\
\hline Sperm DNA fragmentation (\%) & $14.1(10.3-17.9)$ & $23.9(12.9-35.0)$ & 0.038 \\
\hline Age of female (yr) & $33.9(32.9-35.0)$ & $36.1(32.0-40.2)$ & NS \\
\hline No. of cycles & $2(1.6-2.5)$ & $1.9(0.7-3.1)$ & NS \\
\hline Basal serum FSH level (mlU/mL) & $5.7(4.9-6.5)$ & $7.3(5.9-8.8)$ & 0.015 \\
\hline Random serum AMH level (ng/mL) & $3.8(2.7-4.8)$ & $1.9(0.6-3.2)$ & NS \\
\hline Dose of gonadotropin (ampule) ${ }^{\mathrm{a})}$ & $21.8(20.0-23.7)$ & $24.5(20.1-28.9)$ & NS \\
\hline Peak serum estradiol level (pg/mL) & $1,941(1,450-2,431)$ & $1,893(732-3,053)$ & NS \\
\hline No. of mature oocytes & $7.6(6.0-9.2)$ & $4.3(1.6-7.0)$ & 0.025 \\
\hline \multicolumn{4}{|l|}{ Method of insemination } \\
\hline IVF & 28 & 5 & NS \\
\hline ICSI & 16 & 5 & \\
\hline Split insemination & 1 & 0 & \\
\hline Fertilization rate (\%) & $83(77.3-88.7)$ & $76.9(58.9-95.0)$ & NS \\
\hline No. of day 3 transfers & 32 & 8 & NS \\
\hline No. of day 5 transfers & 13 & 2 & \\
\hline No. of embryos transferred & $2(1.8-2.2)$ & $1.6(1.1-2.1)$ & NS \\
\hline \multicolumn{4}{|l|}{ Embryo quality (day 3 transfer only) } \\
\hline No. of grade $A$ & $1(0.4-1.6)$ & $1(0.9-1.5)$ & NS \\
\hline No. of grades $A+B$ & $2(1.7-2.2)$ & $1(0.9-1.8)$ & NS \\
\hline Endometrial thickness (mm) & $8.8(8.3-10.0)$ & $10.6(8.0-16.0)$ & NS \\
\hline Triple pattern endometrium (\%) & 95.5 & 90 & NS \\
\hline
\end{tabular}

Values are presented as median (95\% confidence interval). Mann-Whitney $U$-test.

NS, not significant; FSH, follicle-stimulating hormone; AMH, anti-Müllerian hormone; IVF, in vitro fertilization; ICSI, intracytoplasmic sperm injection.

a)Each ampule contains 75 IU of gonadotropin.

Table 4. Predictive power of various factors for miscarriage

\begin{tabular}{llllcccccc}
\hline Variable & Cutoff & AUC & $95 \% \mathrm{Cl}$ & Sensitivity & Specificity & $+\mathrm{LR}$ & $-\mathrm{LR}$ & $+\mathrm{PV}$ & $-\mathrm{PV}$ \\
\hline Sperm DNA fragmentation $(\%)$ & $>13$ & $0.713^{\mathrm{a})}$ & $0.575-0.827$ & 80.0 & 62.2 & 2.12 & 0.32 & 32.0 & 93.3 \\
Basal serum FSH level (mlU/mL) & $>6.7$ & $0.768^{\mathrm{a})}$ & $0.614-0.883$ & 77.8 & 79.4 & 3.78 & 0.28 & 50.0 & 93.1 \\
No. of mature oocytes & $\leq 3$ & $0.733^{\mathrm{a})}$ & $0.597-0.843$ & 70.0 & 77.8 & 3.15 & 0.39 & 41.2 & 92.1 \\
Age of male partner (yr) & $>36$ & 0.570 & $0.429-0.703$ & 70.0 & 53.3 & 1.50 & 0.56 & 25.0 & 88.9 \\
Age of female (yr) & $>35$ & 0.622 & $0.481-0.749$ & 60.0 & 73.3 & 2.25 & 0.55 & 33.3 & 89.2
\end{tabular}

$\mathrm{AUC}$, area under the curve; $\mathrm{Cl}$, confidence interval; $+\mathrm{LR}$, positive likelihood ratio; - $\mathrm{LR}$, negative likelihood ratio; $+\mathrm{PV}$, positive predictive value; $-\mathrm{PV}$, negative predictive value; $\mathrm{FSH}$, follicle-stimulating hormone.

a) $p<0.05$.

lower (Table 3).

A ROC analysis revealed that the cutoff values for predicting miscarriage were an SDF level $>13 \%$, a basal serum FSH level $>6.7 \mathrm{mlU}$ / $\mathrm{mL}$, and $\leq 3$ mature oocytes; all three cutoff values were statistically significant (Table 4). Neither the male partner's age nor female age was a predictor of miscarriage. However, since both the male partner's age and female age were possible confounders, multiple regression analysis was performed, including five parameters (SDF level, basal serum FSH level, number of mature oocytes, the male partner's age, and female age). SDF was found to be the only significant predictive factor of miscarriage (odds ratio [OR], 1.051; 95\% Cl, 1.001-1.104).

\section{IVF outcomes according to SDF and ovarian responsiveness}

Cycles with three or fewer mature oocytes usually indicate POR. Since high SDF levels ( $>13 \%$ ) and POR were significant predictive factors of miscarriage, we divided the whole population into a low- 
Table 5. Clinical outcomes according to the SDF and ovarian responsiveness

\begin{tabular}{|c|c|c|c|c|c|c|}
\hline \multirow{2}{*}{ Variable } & \multicolumn{2}{|c|}{ SDF $\leq 13 \%$} & \multirow{2}{*}{$p$-value } & \multicolumn{2}{|c|}{ SDF $>13 \%$} & \multirow{2}{*}{$p$-value } \\
\hline & $\operatorname{NOR}(n=58)$ & $\operatorname{POR}(n=36)$ & & $\operatorname{NOR}(n=35)$ & $\operatorname{POR}(n=40)$ & \\
\hline Age of male partner (yr) & $37(36.2-38.5)$ & $36(35.8-39.0)$ & NS & $37(36.6-39.5)$ & $41(39.5-42.6)$ & 0.010 \\
\hline Age of female (yr) & $34(33.6-35.8)$ & $34.5(34.1-36.6)$ & NS & $35(34.4-36.7)$ & $39(37.6-40.2)$ & 0.001 \\
\hline Basal serum FSH level (mlU/mL) & $4.9(4.7-5.7)$ & $6.6(5.5-8.1)$ & 0.012 & $5.9(4.9-6.9)$ & $8.1(6.9-10.2)$ & 0.007 \\
\hline Random serum AMH level (ng/mL) & $3.6(3.1-4.7)$ & $0.8(0.7-2.5)$ & 0.001 & $2.6(2.4-4.2)$ & $1.1(1.1-1.8)$ & 0.001 \\
\hline Peak serum estradiol level (pg/mL) & $1,998(1,934-2,801)$ & $698(719-1,452)$ & 0.001 & $1,942(1,526-2,502)$ & $651(651-1,106)$ & 0.001 \\
\hline No. of mature oocytes & $8(7.9-10.1)$ & $2(1.6-2.2)$ & 0.001 & $6(5.9-8.2)$ & $2(1.7-2.2)$ & 0.001 \\
\hline No. of day 3 transfers & 38 & 29 & 0.001 & 24 & 38 & 0.002 \\
\hline No. of day 5 transfers & 19 & 0 & & 9 & 0 & \\
\hline No. of ET canceled cycles & 1 & 7 & 0.009 & 2 & 2 & NS \\
\hline No. of embryos transferred & $2(2.0-2.3)$ & $1(1.3-1.9)$ & 0.001 & $2(1.9-2.3)$ & $2(1.6-2.1)$ & NS \\
\hline \multicolumn{7}{|l|}{ Embryo quality (day 3 transfer only) } \\
\hline No. of grade $A$ & $1(1.1-1.6)$ & $1(0.4-1.0)$ & 0.034 & $1(0.7-1.3)$ & $1(0.4-0.9)$ & NS \\
\hline No. of grade $A+B$ & $2(1.9-2.4)$ & $1(1.0-1.6)$ & 0.002 & $2(1.5-2.2)$ & $1(1.3-1.7)$ & NS \\
\hline Clinical pregnancy per transfer $(\%, n)$ & $40.3(23 / 57)$ & $24.1(7 / 29)$ & NS & $45.4(15 / 33)$ & $26.3(10 / 38)$ & NS \\
\hline Miscarriages per clinical pregnancy $(\%, n)$ & $4.3(1 / 23)$ & $14.2(1 / 7)$ & NS & $13.3(2 / 15)$ & $60.0(6 / 10)$ & 0.045 \\
\hline
\end{tabular}

Values are presented as median ( $95 \%$ confidence interval). Mann-Whitney $U$-test.

SDF, sperm DNA fragmentation; NOR, normal ovarian response ( $>3$ mature oocytes); POR, poor ovarian response ( $\leq 3$ mature oocytes); FSH, follicle-stimulating hormone; $\mathrm{AMH}$, anti-Müllerian hormone; $\mathrm{ET}$, embryo transfer.

SDF ( $\leq 13 \%)$ and high-SDF (>13\%) group and further divided them into POR and normal ovarian response (NOR) groups (Table 5). In the low-SDF group, the miscarriage rate was similar between the POR and NOR groups (14.2\% vs. 4.3\%). However, in the high-SDF group, the miscarriage rate was significantly higher in the POR group than in the NOR group $(60.0 \%$ vs. $13.3 \%, p=0.045)$. The pregnancy rate did not differ across the four sub-groups.

\section{Discussion}

In the present study, SDF levels did not affect the fertilization rate or pregnancy rate in IVF/ICSI cycles, but did significantly affect the miscarriage rate. We found that the miscarriage rate was also affected by POR, which is generally considered to be a poor prognostic factor. In this study, the pregnancy rate was significantly lower ( $25.4 \%$ vs. $42.2 \%, p=0.04)$ and the miscarriage rate was significantly higher $(41.2 \%$ vs. $7.9 \%, p=0.01)$ in the POR group than in the NOR groups (Table 5). Nonetheless, multiple regression analysis revealed that SDF was the only significant predictive factor of miscarriage.

The miscarriage rate was highest in POR patients with a high SDF level (60\%); this rate was significantly higher than that of NOR patients with a low SDF level (4.3\%) or NOR patients with a high SDF level (13.3\%). This indicates that high SDF levels only contributed to miscarriage in the POR group. Therefore, SDF testing may be of particular clinical significance for couples with POR.

When evaluating the effects of male parameters on IVF outcomes, it is important to control for female parameters, such as age, ovarian reserve, and the number of retrieved oocytes. Dar et al. [9] evaluated the influence of high SDF levels on fertilization, clinical pregnancy, and miscarriage rates. Couples were matched by female age and serum anti-Müllerian hormone level since these variables could act as potential confounders. They showed that the fertilization and clinical pregnancy rates were similar between groups with a high SDF level $(>50 \%)$ and groups with a low SDF level $(\leq 15 \%)$. They showed a trend for a higher miscarriage rate in the high SDF group, but it did not reach statistical significance.

Jin et al. [10] evaluated the effect of SDF levels on IVF outcomes according to ovarian reserve. Reduced ovarian reserve was defined as basal FSH $>10 \mathrm{mIU} / \mathrm{mL}$, an antral follicle count $<6$, and a female age $\geq 38$ years. They showed that SDF levels only had a significant impact on the clinical pregnancy and live-birth rates among women with reduced ovarian reserve; in women with normal ovarian reserve, SDF levels had no impact.

In fact, disagreements exist about the association between SDF levels and miscarriage in IVF cycles. Two meta-analyses reported that high SDF levels were associated with higher miscarriage rates $[4,5]$, but no association was reported in a recent meta-analysis [6]. The reasons for this disparity are largely unknown, but the use of different types of assays to assess DNA damage may be a reason. Robinson et al. [4] found that the association between high SDF levels and miscarriage was strongest when using the TUNEL assay. The TUNEL assay directly quantifies DNA damage by the incorporation of labeled dUTP into single- and double-stranded DNA breaks. It is generally known to have a higher sensitivity and specificity for the detec- 
tion of SDF than the sperm chromatin dispersion (SCD) test or sperm chromatin structure assay (SCSA). A comprehensive study reported that the cutoff value for defining male factor infertility was $20.1 \%$ with a sensitivity of 0.764 and specificity of 0.952 when TUNEL analysis was applied [11].

Although an earlier meta-analysis reported no association between SDF levels and the fertilization rate, conflicting results have been reported on this topic [3]. High SDF levels have been reported to exert a negative effect on the fertilization rate, but studies have also found no association [9,12-17]. Some studies have reported a negative impact in standard IVF cycles but no impact in ICSI cycles $[16,18]$. In the current study, no associations between SDF levels and the fertilization rate were found in the whole population or in the five subgroups divided according to the insemination method, indication of ICSI, and semen quality.

It has been reported that DNA-damaged sperm cells could fertilize the oocyte regardless of the degree of DNA damage, but that embryonic development and early pregnancy loss were closely related to the degree of damage [19]. Such a finding explains the absence of an association between SDF levels and the fertilization rate. However, high SDF levels have been reported to have an adverse impact on embryogenesis. Tesarik et al. [20] showed that high SDF levels were associated with a "late paternal effect" during the activation of male gene expression. Sperm cells with highly damaged DNA caused certain paternal genome deficiencies and defective genomic activation within the embryo, thereby potentially exerting detrimental effects on late embryonic development [21,22].

The underlying mechanisms behind the close association between higher SDF levels and higher miscarriage rates in the POR group are largely unknown and require further investigation. Oocytes have been reported to possess the capability to repair damaged sperm DNA in murine models [19], and it was suggested that the effect of SDF levels on IVF/ICSI outcomes depended on oocyte quality [23]. The oocyte quality in the POR group might have been poor, meaning that oocytes in the POR group might have been less capable of repairing damaged sperm DNA than those in the NOR group.

The limitations of this study include the retrospective nature of the study design and small sample size. We used the TUNEL assay for SDF measurements, and therefore, a direct comparison with SDF assessed by SCD or SCSA was not possible.

In conclusion, no association was found between SDF levels and the fertilization rate or pregnancy rate in IVF/ICSI cycles. However, the SDF level significantly affected the miscarriage rate, especially in women with POR. These findings indicate that SDF testing should be performed in couples with POR to provide additional information on the prognosis of pregnancy.

\section{Conflict of interest}

No potential conflict of interest relevant to this article was reported.

\section{References}

1. World Health Organization. WHO laboratory manual for the examination and processing of human semen. 5th ed. Geneva: World Health Organization; 2010.

2. Practice Committee of the American Society for Reproductive Medicine. The clinical utility of sperm DNA integrity testing: a guideline. Fertil Steril 2013;99:673-7.

3. Li Z, Wang L, Cai J, Huang H. Correlation of sperm DNA damage with IVF and ICSI outcomes: a systematic review and meta-analysis. J Assist Reprod Genet 2006;23:367-76.

4. Robinson L, Gallos ID, Conner SJ, Rajkhowa M, Miller D, Lewis S, et al. The effect of sperm DNA fragmentation on miscarriage rates: a systematic review and meta-analysis. Hum Reprod 2012; 27:2908-17.

5. Zhao J, Zhang Q, Wang Y, Li Y. Whether sperm deoxyribonucleic acid fragmentation has an effect on pregnancy and miscarriage after in vitro fertilization/intracytoplasmic sperm injection: a systematic review and meta-analysis. Fertil Steril 2014;102:9981005.e8.

6. Zhang Z, Zhu L, Jiang H, Chen H, Chen Y, Dai Y. Sperm DNA fragmentation index and pregnancy outcome after IVF or ICSI: a meta-analysis. J Assist Reprod Genet 2015;32:17-26.

7. Osman A, Alsomait H, Seshadri S, El-Toukhy T, Khalaf Y. The effect of sperm DNA fragmentation on live birth rate after IVF or ICSI: a systematic review and meta-analysis. Reprod Biomed Online 2015;30:120-7.

8. Kim SK, Jee BC, Kim SH. Histone methylation and acetylation in ejaculated human sperm: effects of swim-up and smoking. Fertil Steril 2015;103:1425-31.

9. Dar S, Grover SA, Moskovtsev SI, Swanson S, Baratz A, Librach CL. In vitro fertilization-intracytoplasmic sperm injection outcome in patients with a markedly high DNA fragmentation index (>50\%). Fertil Steril 2013;100:75-80.

10. Jin J, Pan C, Fei Q, Ni W, Yang X, Zhang L, et al. Effect of sperm DNA fragmentation on the clinical outcomes for in vitro fertilization and intracytoplasmic sperm injection in women with different ovarian reserves. Fertil Steril 2015;103:910-6.

11. Ribas-Maynou J, Garcia-Peiro A, Fernandez-Encinas A, Abad C, Amengual $\mathrm{MJ}$, Prada $\mathrm{E}$, et al. Comprehensive analysis of sperm DNA fragmentation by five different assays: UNEL assay, SCSA SCD test and alkaline and neutral Comet assay. Andrology 2013; 1:715-22. 
12. Lin MH, Kuo-Kuang Lee R, Li SH, Lu CH, Sun FJ, Hwu YM. Sperm chromatin structure assay parameters are not related to fertilization rates, embryo quality, and pregnancy rates in in vitro fertilization and intracytoplasmic sperm injection, but might be related to spontaneous abortion rates. Fertil Steril 2008;90:352-9.

13. Velez de la Calle JF, Muller A, Walschaerts M, Clavere JL, Jimenez $C$, Wittemer $C$, et al. Sperm deoxyribonucleic acid fragmentation as assessed by the sperm chromatin dispersion test in assisted reproductive technology programs: results of a large prospective multicenter study. Fertil Steril 2008;90:1792-9.

14. Chi HJ, Chung DY, Choi SY, Kim JH, Kim GY, Lee JS, et al. Integrity of human sperm DNA assessed by the neutral comet assay and its relationship to semen parameters and clinical outcomes for the IVF-ET program. Clin Exp Reprod Med 2011;38:10-7.

15. Esbert M, Pacheco A, Vidal F, Florensa M, Riqueros M, Ballesteros $A$, et al. Impact of sperm DNA fragmentation on the outcome of IVF with own or donated oocytes. Reprod Biomed Online 2011; 23:704-10.

16. Simon L, Brunborg G, Stevenson M, Lutton D, McManus J, Lewis SE. Clinical significance of sperm DNA damage in assisted reproduction outcome. Hum Reprod 2010;25:1594-608.

17. Wang M, Sun J, Wang L, Gao X, Lu X, Wu Z, et al. Assessment of density gradient centrifugation (DGC) and sperm chromatin dis- persion (SCD) measurements in couples with male factor infertility undergoing ICSI. J Assist Reprod Genet 2014;31:1655-63.

18. Pregl Breznik B, Kovacic B, Vlaisavljevic V. Are sperm DNA fragmentation, hyperactivation, and hyaluronan-binding ability predictive for fertilization and embryo development in in vitro fertilization and intracytoplasmic sperm injection? Fertil Steril 2013; 99:1233-41.

19. Ahmadi A, Ng SC. Fertilizing ability of DNA-damaged spermatozoa. J Exp Zool 1999;284:696-704.

20. Tesarik J, Greco E, Mendoza C. Late, but not early, paternal effect on human embryo development is related to sperm DNA fragmentation. Hum Reprod 2004;19:611-5.

21. Sakkas D, D’Arcy Y, Percival G, Sinclair L, Afnan M, Sharif K. Use of the egg-share model to investigate the paternal influence on fertilization and embryo development after in vitro fertilization and intracytoplasmic sperm injection. Fertil Steril 2004;82:74-9.

22. Seli E, Gardner DK, Schoolcraft WB, Moffatt O, Sakkas D. Extent of nuclear DNA damage in ejaculated spermatozoa impacts on blastocyst development after in vitro fertilization. Fertil Steril 2004;82:378-83.

23. Meseguer M, Santiso R, Garrido N, Garcia-Herrero S, Remohi J, Fernandez JL. Effect of sperm DNA fragmentation on pregnancy outcome depends on oocyte quality. Fertil Steril 2011;95:124-8. 